Endocrinol. Japon. 1989, 36 (2), 269-274

\title{
ACTH Determination in a Petrosal Sinus Venous Specimen after Corticotrophin Releasing Factor Provides the Best Clue on the Laterality of Microadenoma in Cushing's Disease
}

\author{
Shozo YAMADA ${ }^{1)}$, Yoshimasa SHISHIBA ${ }^{2)}$, Shinzi SAWANO ${ }^{2)}$ \\ And Tadashi AIBA ${ }^{1)}$ \\ Division of Neurosurgery ${ }^{1)}$ and Division of Endocrinology $y^{2)}$ Toranomon \\ Hospital and Okinaka Memorial Institute of Medical Research
}

\begin{abstract}
A 42-year-old woman with Cushing's disease was endocrinologically cured after transsphenoidal selective excision of a left-sided microadenoma. Simultaneous samplings from the bilateral inferior petrosal sinuses with ovine corticotrophin-releasing factor (CRF) stimulation were performed preoperatively to evaluate some relations between the localization of a possible microadenoma and the levels of ACTH in inferior petrosal sinuses. The data for the venous samplings were as follows:-(1) The basal levels of ACTH obtained simultaneously from both inferior petrosal sinuses and peripheral vein were about the same. (2) A significant difference, with a high level of ACTH from the ipsilateral side of the microadenoma, was demonstrated after CRF stimulation. (3) The magnitude of increase in ACTH on the contralateral side after CRF was similar to that of the peripheral response.

It is recommended that all patients with ACTH dependent Cushing's syndrome and negative radiological findings should have bilateral simultaneous inferior petrosal venous sampling with CRF stimulation.
\end{abstract}

Selective venous sampling from the inferior petrosal sinus has been reported as a reliable and useful method to establish the presence and laterality of ACTH-secreting microadenomas (Corrigan et al., 1977; Findling et al., 1981; Drury et al., 1982; Manni et al., 1983; Doppman et al., 1984. Oldfield et al., 1985; Zovickian et al., 1988). However, there is still uncertainty regarding

Received December 16, 1988

Mail all correspondence to:

Dr. SHOZO YAMADA; Division of Neurosurgery Toranomon Hospital, 2-2-2 Toranomon Minato$\mathrm{ku}$, Tokyo 105 the interpretation of the data obtained by this technique (Chrousos et al., 1985; Cuneo et al., 1985; Kageyama et al., 1985 ; Lüdeck 1988). Lately some modifications have been developed to improve the efficacy of this technique (Cuneo et al., 1985; Lüdeck 1988). Bilateral simultaneous inferior petrosal venous sampling with corticotrophin releasing factor (CRF) stimulation is one of such innovations (Chrousos et al., 1985; Hauffa et al., 1986; Landolt et al., 1986). In order to further document the efficacy of this technique, the following case is presented with brief discussion. 


\section{Case Report}

This 42-year-old woman was referred to us for evaluation of hypertension and hypercorticolism. A diagnosis of Cushing's disease was made based on the following endocrinological data: plasma cortisol levels were high $(25-27 \mu \mathrm{g} / \mathrm{dl})$ with loss of circadian rhythm; 24 hour urinary excretion of 17-hydroxycorticosteroid (17-OHCS) and 17-ketosteroid (17-KS) was increased $(24.7$
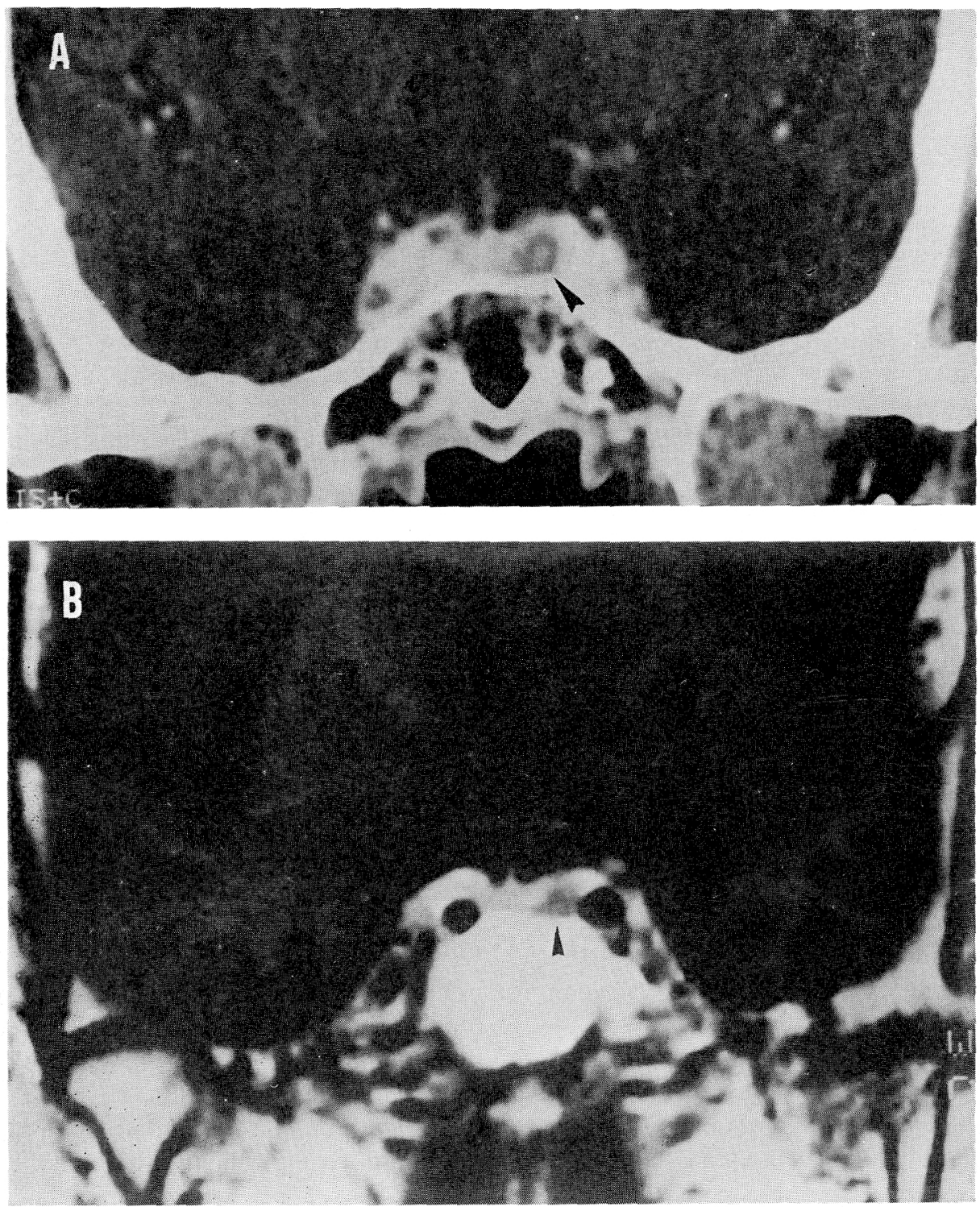

Fig. 1. Direct coronary enhanced CT scan (A) and $T_{1}$-weighted coronary $M R$ image with Gd-DTPA (B) showing hypodensity (arrow head) and hypointensity (arrow head), respectively, on the left side which represent microadenoma verified at surgery. (Gd-DTPA; gadolinium-diethylene triamine pentaacetic acid) 
Table 1. Result of selective sampling for ACTH, cortisol and PRL with CRF stimulation

\begin{tabular}{|c|c|c|c|c|c|c|c|c|c|}
\hline \multirow{3}{*}{$\begin{array}{l}\text { Time after } \\
\text { CRF (min) }\end{array}$} & \multirow{2}{*}{\multicolumn{3}{|c|}{$\begin{array}{c}\text { Peripheral } \\
\text { vein }\end{array}$}} & \multicolumn{6}{|c|}{ Inferior petrosal sinus } \\
\hline & & & & & eft side & & & ght side & \\
\hline & ACTH & cortisol & PRL & ACTH & cortisol & PRL & ACTH & cortisol & PRL \\
\hline Before & 139 & 24.6 & 29.5 & 135 & 24.0 & 35.8 & 136 & 24.4 & 38.2 \\
\hline 5 & 248 & 26.6 & 29.1 & 4930 & 24.5 & 60.1 & 169 & 22.3 & 27.8 \\
\hline 10 & 408 & 29.0 & 28.3 & 3870 & 30.7 & 49.9 & 274 & 29.8 & 30.2 \\
\hline 15 & 468 & 34.0 & 31.2 & 4220 & 38.2 & 61.1 & 386 & 35.8 & 31.1 \\
\hline 20 & 498 & 36.6 & 29.5 & 3410 & 37.5 & 50.3 & 600 & 36.8 & 56.2 \\
\hline 25 & 512 & 38.7 & & 2400 & 39.3 & 48.6 & 422 & 39.4 & 31.8 \\
\hline 35 & 527 & 41.9 & & 803 & 41.2 & 36.0 & 536 & 37.9 & 49.4 \\
\hline
\end{tabular}

ACTH, Cortisol and PRL levels are presented as $\mathrm{pg} / \mathrm{ml}, \mu \mathrm{g} / \mathrm{dl}$ and $\mathrm{ng} / \mathrm{ml}$, respectively.

PRL level at 25 and 35 minutes in peripheral vein could not be measured because of the shortage of samples.

and $10.6 \mathrm{mg} /$ day, respectively.); dexamethasone suppression test was positive (i.e. after $2 \mathrm{mg}$ dexamethasone 6-hourly for 48 hours, urinary excretion of 17-OHCS and 17-KS was suppressed.); plasma ACTH levels ranged from 120 to $139 \mathrm{pg} / \mathrm{ml}$ without circadian rhythm and responded remarkably after CRF administration. A left sided pituitary microadenoma was suggested by both high resolution coronary computerized tomography (CT) and magnetic resonance imaging (MRI). (Fig. 1)

In order to further confirm the diagnosis and localization of the microadenoma preoperatively, the bilateral inferior petrosal venous sampling with CRF stimulation was performed by the Seldinger technique via the femoral vein. The location of the catheter tips in both inferior petrosal sinuses was confirmed before and after CRF stimulation by injecting contrast medium into the catheter. Blood samples were taken simultaneously from both inferior petrosal sinuses and from the antecubital vein before and $5,10,15,20,25$ and 35 minutes after ovine CRF administration (100 $\mu \mathrm{g}$ intravenous bolus injection), and ACTH, cortisol and PRL concentrations were measured by radioimmunoassay. The basal level of ACTH from the left inferior petrosal sinus was not different from that of the right side or the peripheral venous blood. However, after CRF injection, ACTH on the left side increased tremendously. In contrast, the ACTH increase on the right side was much smaller and almost identical with that of the peripheral vein. The cortisol concentration in both the petrosal sinuses and the peripheral vein increased only slightly after CRF but there was no significant difference between them. The ratio of inferior petrosal sinus to peripheral blood concentrations $(\mathrm{C} / \mathrm{P}$ ratio) of PRL ranged from 0.96 to 1.91 (right side) and 1.20 to 2.07 (left side). (Table 1)

At the time of transphenoidal surgery, a $4 \mathrm{~mm}$ microadenoma was verified on the left side and selective adenomectomy resulted in clinical and biological remission of the Cushing's disease.

\section{Discussion}

The differentiation of Cushing's dịsease from ectopic ACTH syndrome and preoperative imaging of a pituitary adenoma are mandatory prior to transsphenoidal surgery. Endocrine testings are also essential but may be inconclusive or even misleading in some cases (Aron et al., 1981; Findling et al., 1985; Grossman et al., 1988). Modern 
radiological examinations have been proven to be most beneficial in the evaluation of pituitary adenomas, but they too have some limitations. Even with very sophisticated technology such as high resolution CT scan and high field-strength MRI, difficulty can be encountered especially in Cushing's disease with small microadenoma (Saris et al., 1987; Nichols et al., 1988).

In these inconclusive cases, inferior petrosal venous sampling has been recommended by many investigators (Chrousos et al., 1985; Kageyama et al., 1985 ; Landolt et al., 1986; Zovickian et al., 1988). Early works on unilateral inferior petrosal sinus and jugular vein sampling (Corrigan et al., 1977; Findling et al., 1981; Drury et al., 1982) have emphasized the importance of bilateral simultaneous inferior petrosal venous sampling which should be performed if more reliance is to be placed on the results (Manni et al., 1983 ; Doppman et al., 1984; Oldfield et al., 1985; Zovickian et al., 1988). It has also been reported subsequently that bilateral inferior venous sampling may give rise to false negatives (Cuneo et al., 1985; Kageyama et al., 1985). Indeed, if the baseline ACTH levels had been only considered in this case, this result would have failed to give any clue in confirming the laterality of the microadema. It has been suggested that these false negatives are often associated in this technique due to low levels of ACTH (Kageyama et al., 1985), anatomical variation of veins (Shiu et al., 1968), irregular bursts of ACTH secretion from the adenoma (Cuneo et al., 1985), unequivalent dilution of pituitary venous blood (Zovickian et al., 1988), and so on. Consequently, simultaneous PRL measurement has been proposed as an indicator of successful sampling of pituitary drainage (Tanaka et al., 1984; Yokoe et al., 1987). The absence of a difference among baseline ACTH levels in both petrosal samples and peripheral sample in this case may therefore due to the possibility of the unsuccessful sampling of pituitary drainage, since the PRL C/P ratio was 1.3 (right side) and 1.2 (left side). Recently, Zovickian et al. (1988), however, have claimed that correction of inferior petrosal sinus ACTH levels by dividing the ACTH concentration from each side by the ratio of the inferior petrosal sinus to peripheral blood levels of PRL, TSH, and $\alpha$ HCG does not improve preoperative localization of ACTH-secreting microadenomas and may lead to incorrect lateralization. Moreover, in this study the PRL C/P ratio was not constant during this procedure, and the maximum PRL $\mathrm{C} / \mathrm{P}$ ratio was 1.9 (right side) and 2.1 (left side). Thus, further investigations should be needed to clarify the significance of simultaneous measurement of other pituitary hormones including PRL, and it is still controversial what kinds of the innovation are more reliable to assure valid sampling results.

Inferior petrosal venous sampling with CRF stimulation has been reported as a new modification by a few authors (Chrousos et al., 1985; Hauffa et al., 1986; Landolt et al., 1986). However, the number of cases investigated by this technique is still small in the literature; six cases reported by Chrousos et al., (1985), eight cases by Landolt et al., (1986) and one case by Hauffa et al., (1986). In most of these cases the site of the adenoma is made apparent on the basis of the prestimulated samples (Chrousos et al., 1985; Landolt et al., 1986). The practical significance of this new technique is therefore still unclear and further experience is still required before it can be generally accepted. We believe the data based on venous sampling obtained from our patient is very instructive and exciting from the viewpoint of the interpretation of bilateral simultaneous inferior petrosal sinus sampling with CRF stimulation, even though it was not essential in this case for the diagnosis and localization of the microadenoma preoperatively. Namely, the baseline ACTH concentrations obtained 
simultaneously from both inferior petrosal sinuses and peripheral vein were almost the same as mentioned previously. However, after CRF stimulation, there were much higher levels of ACTH in the petrosal venous samples from the ipsilateral side of the microadenoma, indicating without any doubt the laterality of the adenoma. This finding corresponds well with those of 2 cases and 1 case reported by Landolt et al., (1986) and Hauffa et al., (1986), respectively. The superiority of this technique may be due to the fact that CRF stimulation artificially induces a secretory phase of ACTH secretion from the adenoma. In contrast, the magnitude of increase in ACTH in the contralateral side of the adenoma was not different from that of the peripheral ACTH response during this procedure. Chrousos et al. (1985) have reported a definite but less marked gradient which can be consistently detected in the contralateral sinus. The magnitude of contralateral response to CRF may depend on the degree the normal ACTH secreting pituicytes are suppressed by the hypercorticolism.

In conclusion, this case clearly demonstrates that bilateral inferior petrosal venous sampling with CRF stimulation is superior to the conventional technique without CRF stimulation to identify the side of the microadenoma preoperatively, and it is possible, taking into account the great differences between ACTH levels in the bilateral petrosal venous samples induced by CRF stimulation, that petrosal venous sampling may be replaced by jugular vein sampling which is technically much easier. Futhermore, we would like to suggest that inferior petrosal venous sampling with CRF stimulation should be performed bilaterally even for the purpose of differentiating Cushing's disease from other causes of ACTH dependent Cushing's syndrome.

\section{Acknowledgements}

The authors are indebted to Dr. Y. Imai and Miss Y. Daimon for their collaboration throughout the study.

\section{References}

Aron, D. C., J. B. Tyrell, P. A. Fitzgerald, J. W. Findling and P. H. Forsham (1981). Cushing's syndrome; problems in diagnosis. Medicine 60, 25-35.

Chrousos, G.P., T.H. Schuermeyer, J. Doppman, E. H. Oldfield, H. M. Schulte, P. W. Gold and D. L. Loriaux (1985). Clinical application of corticotrophin-releasing factor. Ann. Intern Med. 102, 344-358.

Corrigan, D. F., M. Schaaf, R. A. Whaley, C. L. Czerwinski and J. M. Earll (1977). Selective venous sampling to differentiate ectopic ACTH secretion from pituitary Cushing's syndrome. N. Engl. J.Med. 296, 861-862.

Cuneo, R., D. Ross, M. MacFarlane, E. A. Espiner and R. A. Donald (1985). Sequential inferior petrosal venous sampling for Cushing's disease. N. Engl. J. Med. 313, 582.

Doppman, J. L., E. M. Oldfield, A. G. Krudy, G. P. Chrousos, H. M. Schulte, M. Schaaf and D. L. Loriaux (1983). Petrosal sinus sampling for Cushing Syndrome : anatomical and technical considerations. Radiology 150, 99-103.

Drury, P. L., S. Ratter, S. Tomlin, J. Williams, J. E. Dacie, L. H. Rees and G. M. Besser (1982). Experience with selective venous sampling in diagnosis of ACTH-dependent Cushing's syndrome. Br. Med. J. 284, 9-12. Findling, J. W., D. C. Aron, J. B. Tyrrel1, J. H. Sinsako, P. A. Fitzgerald, D. Norman, C. B. Wilson and P. H. Forsham (1981). Selective venous sampling for ACTH in Cushing's syndrome. Ann. Intern. Med. 94, 647-652.

Findling, J. W., D. C. Aron and J. B. Tyrrell (1985). Cushing's disease. In the pituitary gland (ed A. Imura). Paven Press, New York. 411-466.

Grossman, A. B., T. A. Howlett, L. Perry, D. H. Coy, M. O. Savage, P. Lavender, L. H. Rees and G. M. Besser (1988). CRF in the 
differential diagnosis of Cushing's syndrome : a comparison with the dexamethason suppression test. Clin. Endocrinol. 29, 167-178.

Hauffa, B. P., H. Stolecke and H. M. Schulte (1986). Cushing disease; successful preoperative lateralization of an ACTH-producing pituitary microadenoma by simultaneous bilateral inferior petrosal venous sampling with carticotrophin-releasing hormone stimulation. Eur. J. Pediatr. 145, 559-562.

Kageyama, N., A. Kuwayama, T. Takahashi, M. Negoro and K. Ichihara (1985). Diagnosis, treatment and postoperative results of Cushing's disease. Neurosurg. Rev. 8, 177-183.

Landolt, A. M., A. Valavanis, J. Girard and A. N. Eberle (1986). Corticotrophin-releasing factor-test used with bilatera1, simultaneous blood-sampling for the diagnosis of pituitarydependent Cushing's disease. Clin. Endocrinol. 25, 687-696.

Lüdecke, D. K. (1988). Localization of microadenomas in Cushing's disease by intraoperative ACTH-RIA. Acta. Endocrinol. 117, 61-62.

Manni, A., R. F. Latshaw, R. Page and R. J. Santen (1983). Simultaneous bilateral venous sampling for adrenocorticotropin in pituitarydependent Cushing's disease: evidence for lateralization of pituitary venous drainage. $J$. Clin. Endocrinol. Metab. 57, 1070-1073.

Nichols, D. A., E. R. Laws, Jr., O. W. Houser and C. F. Abboud (1988). Comparison of magnetic resonance imaging and computed tomography in the preoperative evaluation of pituitary adenoma. Neurosurgery 22, 380385.
Oldfield, E. H., G. P. Chrousos, H. M. Schulte, M. Schaaf, P. E. McKeever, A. G. Krudy, G. B. Cutler, D. L. Loriaux and J. L. Doppman (1985). Preoperative lateralization of ACTHsecreting pituitary microadenomas by bilateral and simultaneous inferior petrosal venous sinus sampling. N. Engl. J. Med. 312, 100 103.

Saris, S. C., N. J. Patronas, J. L. Doppman, D. L. Loriaux, G. B. Cutler, L. K., Nieman, G. P. Chrousos and E. H. Oldfield (1987). Cushing syndrome: pituitary CT scanning. Radiology 162, 775-777.

Shiu, P. C., W. N. Hanaffe, G. H. Wilson and R. W. Rand (1968). Cavernous sinus venography. Am. J. Roentgenol. 104, 57-62.

Tanaka, K., N. Shimizu, M. Nagata, T. Seki and J. Nagai (1984). Identification of source of excessive ACTH production by improved selective venous sampling: simultaneous assay of PRL as a pituitary marker. Endocrinol. Japon. 31, 195-200.

Yokoe, S., M. Negoro, T. Nakane and A. Kuwayama (1987). The significance of ACTH measurement in bilateral petrosal sinus in Cushing's disease : as a method for differential and localizing diagnosis of microadenoma. In Proceedings of the sixth workshop on Pituitay Tumors (Tokyo) edited by K. Shizume, 3136. (in Japanese)

Zovickian, J., E. H. Oldfield, J. L. Doppman, G. B. Cutler Jr. and D. L. Loriaux (1988). Usefulness of inferior petrosal sinus venous endocrine markers in Cushing's disease. $J$. Neurosurg. 68, 205-210. 